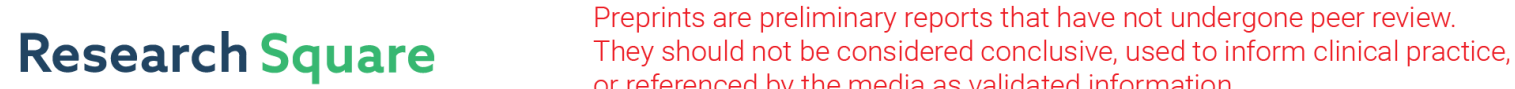 or referenced by the media as validated information. \\ Frequency of hypothyroidism after cardiac catheter in infants with congenital heart diseases: A single center prospective study
}

\section{Boshra Elbaz}

Mansoura University Faculty of Medicine

Hala Elmarsafawy

Mansoura University Faculty of Medicine

Wafaa Laimon ( $\nabla$ wafaalaimon@mans.edu.eg )

Mansoura University Faculty of Medicine https://orcid.org/0000-0003-2807-2412

\section{Research Article}

Keywords: Hypothyroidism, Congenital heart diseases, iodinated contrast media, Cardiac catheter, fluoroscopy

Posted Date: December 17th, 2021

DOI: https://doi.org/10.21203/rs.3.rs-1158782/v1

License: (c) (i) This work is licensed under a Creative Commons Attribution 4.0 International License. Read Full License 


\section{Abstract}

This study aims to determine the incidence, potential onset and clinical course of hypothyroidism following cardiac catheter (CC) in infants with congenital heart diseases (CHD) and to evaluate the predictors for hypothyroidism in this vulnerable group. This prospective study included 102 patients with $\mathrm{CHD}$, aged $\leq 3$ years who underwent $\mathrm{CC}$. Thyroid function tests were assessed before CC, one day, one week, two weeks and four weeks after the procedure. $12 \%$ of the studied group showed hypothyroidism four weeks after CC. Univariate analysis revealed that the significant predictors of hypothyroidism following $C C$ are aortic stenosis ( $R R=10.0$ (1.49-66.99), $P=0.018)$, duration of fluoroscopy ( $R R=1.12$ (0.991.26), $P=0.05)$, and total cumulative dose of iodinated contrast media (iCM) ( $R R=1.01$ (1.003 -1.01), $P=0.019)$. Multivariate analysis revealed that iCM cumulative dose was the only significant predictor of developing hypothyroidism ( $R R=1.00$ (1.00-1.01), $P=0.04)$. ROC curve analysis showed that the cut-off point of iCM dose for prediction of hypothyroidism evolution is $8.7 \mathrm{gm} / \mathrm{kg}(26.1 \mathrm{ml} / \mathrm{kg}$ ), (sensitivity: $83.3 \%$, specificity: $65.1 \%$ ), while the cut-off point of fluoroscopy duration which predicts development of hypothyroidism is 24 minutes, (sensitivity: $83.3 \%$, specificity: $65.9 \%$ ). In a median follow-up duration of 20 months, acquired hypothyroidism after CC persists in $5 \%$ of this cohort.

Conclusion: Exposure to higher dose of iCM and longer duration of fluoroscopy during $\mathrm{CC}$ are risk factors for evolution of hypothyroidism. We recommend assessment of thyroid profile 4 weeks after CC particularly in patients who received a dose of iCM higher than $8.7 \mathrm{gm} / \mathrm{kg}$ and/or exposed to fluoroscopy for more than 24 minutes.

\section{What Is Known}

- The use of excess iodine leads to transient inhibition of thyroid hormones biosynthesis via the WolffChaikoff effect.

- Infants with congenital heart diseases (CHD) are more prone to hypothyroidism due to higher frequency of abnormal thyroid morphology and routine exposure to supraphysiological doses of iodine.

\section{What Is New?}

- Exposure to a cumulative dose of iodinated contrast media more than $8.7 \mathrm{gm} / \mathrm{kg}$ and a fluoroscopy duration more than $\mathbf{2 4}$ minutes are risk factors for evolution of thyroid hypofunction following cardiac catheter.

- In a median follow-up duration of 20 months, acquired hypothyroidism after $\mathrm{CC}$ persists in $5 \%$ of this cohort.

\section{Introduction}


Thyroid hormone is crucial for brain development and functions in young infants, and even transient hypothyroidism in this age group can lead to neurocognitive deficits [1].

The use of excess iodine leads to transient inhibition of thyroid hormones biosynthesis via the WolffChaikoff effect but, the thyroid gland can escape this inhibitory effect of the high intra-glandular iodine after 48 hours via an auto-release mechanisms. However, the immaturity of this escape phenomenon in young infants render them more vulnerable to develop hypothyroidism following exposure to large doses of iodine [2]. Also, using ionizing radiation even at low doses increases the risk of thyroid hypofunction [5].

Infants with congenital heart diseases (CHD) are more prone to hypothyroidism due to higher frequency of abnormal thyroid morphology and routine exposure to supraphysiological doses of iodine [3]. The sources of excess iodine in those infants are either iodinated contrast media (iCM) during computed tomography angiography (CT-A) and cardiac catheterization (CC), or topical iodine containing antiseptics used for disinfection [2, 4]. Furthermore, infants undergoing CC are exposed to variable doses of ionizing radiation which are usually higher during complex procedures [6].

Hypothyroidism can lead to left ventricular hypertrophy, diastolic dysfunction, increased systemic vascular resistance, and reduced cardiac contractility [3].

Although normal thyroid hormone levels are fundamental for proper cardiac functions, thyroid hormonal assay is not routinely monitored in CHD patients undergoing CC. Few studies investigated the frequency of hypothyroidism following iodine exposure during $\mathrm{CC}$ in patients with $\mathrm{CHD}[2,7,8]$, but with no long term follow up data. A single study followed up the patients for a median period of three years after CC and concluded that the frequency of acquired hypothyroidism after iodine overload was $15.4 \%$ and that it was transient in most patients [4]. None of the published studies assessed the effect of ionizing radiation exposure during $\mathrm{CC}$ on evolution of hypothyroidism in infants with $\mathrm{CHD}$.

The aim of the current research work is to determine the incidence, potential onset and clinical course of hypothyroidism following $\mathrm{CC}$ in $\mathrm{CHD}$ patients aged $\leq 3$ years and to evaluate the predictors for development of thyroid hypofunction in this vulnerable group.

\section{Subjects And Methods}

\section{Participants}

This prospective study included all patients $\leq 3$ years of age with CHD who underwent CC in Mansoura University Children Hospital (MUCH), Mansoura, Egypt from August 2018 to February 2020. Patients with a pre-existing thyroid dysfunction, genetic disorders, midline facial defects or renal impairment were excluded.

\section{Methods}


Patients' data (gender, gestational age, birth weight, age at diagnosis of CHD, type of CHD, age at time of $\mathrm{CC}$, number of procedures using iCM, maximal serum creatinine during exposure duration, total cumulative dose of iCM and duration of fluoroscopy during CC) were collected. Catheter Risk Score for Paediatrics (CRISP) which predicts the serious adverse events for individuals undergoing CC, were calculated for all patients [9].

Serum thyroid stimulating hormone (TSH) and free thyroxine (FT4) were measured for all patients using chemiluminescent immunoassay (ElectroChemiLuminescence [ECL], Roche Cobas 6000 analyser (F. Hoffmann-La Roche Ltd. Investor Relations CH-4070 Basel Switzerland); at the following time points: before CC and then, 1 day, one week, two weeks and four weeks after the procedure. These time points were determined depending on the physiological response of thyroid gland to excess iodine. Hypothyroidism was defined as serum TSH > 10 with or without low serum FT4 [10]. Levothyroxine treatment was started in patients with abnormal thyroid profile which did not revert to normal within four weeks after CC.

In the current study, iodine-free antiseptic agent (Kodan®Tinktur forte, Schülke \& Mayr, Norderstedt, Germany) was used in skin disinfection. In our institution, Omnipaque ${ }^{\mathrm{TM}} 300 \mathrm{mg} \mathrm{l} / \mathrm{ml}$ (manufactured by GE Healthcare Ireland, Cork, Ireland) is used as the standard in CT-A and CC. One milliter of Omnipaque ${ }^{\mathrm{TM}}$ 300 contains $647 \mathrm{mg}$ of iohexol (corresponding to $300 \mathrm{mg}$ of iodine) in aqueous solution.

\section{Ethics}

This study was approved by institutional review board of Mansoura faculty of medicine and was in accordance with the 1964 Helsinki declaration and its later amendments. Code number is MS.18.08.233. Informed written consents were obtained from the patients' parents to conduct and publish this research.

\section{Statistical analysis}

Data were analysed using the Statistical Package of Social Science (SPSS) program for Windows (Standard version 21). The normality of data was first tested with one-sample Kolmogorov-Smirnov test. Continuous variables were presented as mean \pm SD (standard deviation) for parametric data and median (range) for non-parametric data. Qualitative data were described using number and percent. Association between categorical variables was tested using Chi-square test while Fischer exact test and Monte Carlo test were used when expected cell count less than 5. Mann-Whitney $U$ test was used to compare 2 independent groups. Significant predictors in the Univariate analysis were entered into regression model using forward Wald method /Enter. Adjusted relative risk and their 95\% confidence interval were calculated. Receiver Operating Characteristic (ROC) curve analysis was carried out to determine the cutoff points of total iCM and duration of fluoroscopy for detection of hypothyroidism following CC. For all the above-mentioned statistical tests done, the threshold of significance is fixed at $5 \%$ level $(P$-value). The results were considered non-significant when the probability of error is more than $5 \%(P>0.05)$ and significant when the probability of error is less than $5 \%(P \leq 0.05)$. The smaller the $P$-value obtained, the more significant are the results. 


\section{Results}

115 patients with $\mathrm{CHD}$ who underwent CC were enrolled in this study. Thirteen patients were excluded due to pre-existing thyroid hypofunction and/or genetic disorders. A total of 102 participants were included: 42 males (41\%) and 60 females (59\%), with median gestational age of 38 (range: $34-40$ ) weeks, and median birth weight of 3 (range: $1.5-4$ ) kilograms. $88 \%$ of the study cohort had congenital acyanotic heart diseases. The median age of diagnosis of CHD was 4 (range: 0.07-30) months and the median age at the time of assessment of thyroid functions was 13.8 (range: 1-36) months. In the studied group, the median cumulative dose of iCM was 5.29 (range: $0.75-15) \mathrm{gm} / \mathrm{kg}$ and the median duration of fluoroscopy exposure was 20 (range: 5-44) minutes. Patients' characteristics are summarized in Table 1.

Thyroid function tests showed thyroid hypofunction in $24 \%$ of patients (two weeks after CC) versus $12 \%$ of patients (four weeks after CC). (Figure 1)

Patients with abnormal thyroid profile one month after CC, showed neither clinical evidence of hypothyroidism, nor thyroid abnormalities in ultrasound examination.

All patients with SH four weeks post-cardiac catheterization, were assigned into one group "hypothyroidism group" ( $n=12)$ and were compared with patients with normal thyroid profile $(n=90)$. This comparison revealed that aortic stenosis frequency was higher in hypothyroidism group (50\%) as compared to euthyroid group (8.8\%), $P=0.029$. Patients who developed hypothyroidism have received significantly higher cumulative dose of iCM, $P=0.007$ and they had a significant longer fluoroscopy exposure time, $P=0.026$, when compared with the euthyroid group. (Table 2 )

Univariate analysis showed that the significant predictors of evolution of hypothyroidism following CC are aortic stenosis ( $R R=10.0$ (1.49-66.99), $P=0.018)$, duration of fluoroscopy ( $R R=1.12$ (0.99-1.26), $P=0.05)$, and total cumulative dose of $\mathrm{iCM}(\mathrm{RR}=1.01$ (1.003 -1.01), $P=0.019)$. Multivariate analysis revealed that dose of iCM was the only significant predictor of developing hypothyroidism in the studied cohort (RR=1.00 (1.00-1.01), $P=0.04)$. (Table 3)

ROC curve analysis has shown that the cut-off point of cumulative dose of iCM for prediction of developing thyroid hypofunction post cardiac catheterization is $8.7 \mathrm{gm} / \mathrm{kg}(26.1 \mathrm{ml} / \mathrm{kg})$, with a sensitivity of $83.3 \%$ and a specificity of $65.1 \%$. (Table 4 , Figure 2 ) In our cohort, the cut-off point of fluoroscopy duration which predicts evolution of hypothyroidism following CC is 24 minutes, with a sensitivity $83.3 \%$ of and a specificity of $65.9 \%$. (Table 4 , Figure 3 )

Levothyroxine was started for the twelve patients with SH. During a mean follow-up duration of 2.25 years, levothyroxine was successfully discontinued in seven patients (58\%) after a mean duration of 6 months. $5 / 12$ patients ( $42 \%$ ) continued to receive long-term levothyroxine for a median period of 20 months (range: 6-36 months).

\section{Discussion}


Recent years have seen rapid development of $\mathrm{CC}$ as a diagnostic and a therapeutic modality for management of CHD [11]. However, little data are available about the potential hazards of exposure to excess iodine and ionizing radiation during $\mathrm{CC}$ on the thyroid functions in patient with CHD.

Treatment of SH is still a matter of debate. However, beneficial effect of levothyroxine therapy on neurocognitive outcomes and reducing cardiometabolic risk factors has been reported [12].

The current prospective study aimed to assess post-cardiac catheter thyroid functions in CHD patients, aged $\leq 3$ years. In this study, incidence of hypothyroidism one month after CC was $12 \%$. However, seven out of these twelve patients had transient thyroid hypofunction. This finding is consistent with previous reports of thyroid dysfunction following $C C[2,4,7,8]$. In a retrospective study conducted on 104 children with CHD (aged from 0-8 years), post-cardiac catheter hypothyroidism was reported in $15.4 \%$ of the studied population and it was transient in 14 out of 16 patients [4]. Thaker et al. [2] and Dechant el al. [8] reported that the incidence of thyroid hypofunction following CC was $25 \%$ and $28.5 \%$, respectively.

The mechanism of hypothyroidism following CC is multifactorial. Excess iodine blocks the sodium iodine co-transporter and inhibits thyroglobulin synthesis (Wolff-Chaikoff effect) [13]. The mature thyroid gland usually escapes this inhibitory effect of iodine within few days. However, repeated exposure to large doses of iodine particularly in young infants leads to failure of this escape phenomenon [2]. In addition to the injected iodine during different procedures, large amount of topical iodine is applied for disinfection but, it is not practically possible to assess its quantity. Moreover, low dose ionizing radiation exposure seems to increase the risk of hypothyroidism [5]. Infants undergoing complex procedures during CC are expected to be exposed to longer durations and larger doses of ionizing radiation.

In previous studies, cumulative dose of iCM, number of interventional procedures, intake of drugs affecting thyroid functions (e.g., amiodarone), impaired renal functions, and intensive care unit stay were identified as risk factors for evolution of hypothyroidism in infants with CHD [2, 7]. In the current study, larger total iCM and longer duration of fluoroscopy were found out to be risk factors for developing thyroid hypofunction following CC. Furthermore, this research work identified a cumulative iCM dose of $8.7 \mathrm{gm} / \mathrm{kg}(26.1 \mathrm{ml} / \mathrm{kg})$ and a duration of fluoroscopy of 24 minutes as predictors of developing SH after CC with a sensitivity of $83 \%$ of and specificity of $65 \%$.

To the best of our knowledge, this is the largest prospective study from a single center which evaluates thyroid functions following $\mathrm{CC}$ in $\mathrm{CHD}$ patients younger than three years with exclusion of genetic disorders which can be associated with thyroid abnormalities. Serial assessment of thyroid function tests adds to the strength of our study. Moreover, this is the first study to assess effect of fluoroscopy duration during $\mathrm{CC}$ on thyroid profile.

The current study is limited by being a single center study and lack of assessment of renal elimination of iCM as urinary iodine was not measured.

\section{Conclusion}


The incidence of hypothyroidism in infants with CHD four weeks following CC is $12 \%$. Assessment of thyroid function is not routinely performed for infants undergoing $\mathrm{CC}$; hence, the true incidence of $\mathrm{SH}$ in this vulnerable group is likely to be more than the estimated incidence in the current study and previous research work. Exposure to higher dose of $\mathrm{iCM}$ and longer duration of fluoroscope during $\mathrm{CC}$ are risk factors for evolution of thyroid hypofunction. In the light of this study results, we recommend assessment of thyroid profile 4 weeks after CC particularly in patients who received a dose of iCM higher than 8.7 $\mathrm{gm} / \mathrm{kg}$ and/or exposed to fluoroscope for more than $24 \mathrm{~min}$.

\section{Abbreviations}

\section{CHD}

Congenital heart diseases

\section{iCM}

lodinated contrast media

CT-A

computed tomography angiography

CC

Cardiac catheterization

TSH

Thyroid stimulating hormone

\section{FT4}

Free thyroxine

ECL

Electrochemiluminescence

ROC

Receiver Operating Characteristic

$\mathrm{SH}$

Subclinical hypothyroidism

\section{Declarations}

Funding: There is no funding source.

Conflict of Interest: The authors declare that they have no conflict of interest.

Availability of data and material: All data are available from the corresponding author on reasonable request.

Code availability: The clinical data were analysed using the Statistical Package of Social Science (SPSS) program for Windows (Standard version 21). 
Authors Contributors' Statement: BE collected patients' samples, collected, and analysed the data. HE revised and edited the manuscript. WL initiated the main study idea, interpreted thyroid profiles, analysed the data and wrote the first draft of the manuscript. All authors approved the final manuscript as submitted and agree to be accountable for all aspects of the work in ensuring that questions related to the accuracy or integrity of any part of the work are appropriately investigated and resolved.

Ethical approval: This report has been approved by institutional review board and was in accordance with the 1964 Helsinki declaration and its later amendments.

Informed consent to participate: Patients' parents signed informed consent regarding participation.

Informed consent for publication: Informed written consents to publish the data have been obtained from the patients' parents.

\section{References}

1. Dezonne RS, Lima FR, Trentin AG, Gomes FC (2015) Thyroid hormone and astroglia: endocrine control of the neural environment. J Neuroendocrinol 27(6):435-445

2. Thaker VV, Galler MF, Marshall AC, Almodovar MC, Hsu HW, Addis CJ, Feldman HA, Brown RS, Levine BS (2017) Hypothyroidism in Infants With Congenital Heart Disease Exposed to Excess lodine. Journal of the Endocrine Society 1(8):1067-1078

3. Lerner RK, Gruber N, Pollak U (2019) Congenital Heart Disease and Thyroid Dysfunction: Combination, Association, and Implication. World journal for pediatric \& congenital heart surgery 10(5):604-615

4. Kubicki R, Grohmann J, Kunz KG, Stiller B, Schwab KO, van der Werf-Grohmann N (2020) Frequency of thyroid dysfunction in pediatric patients with congenital heart disease exposed to iodinated contrast media - a long-term observational study. Journal of pediatric endocrinology \& metabolism: JPEM 33(11):1409-1415

5. Cioffi DL, Fontana L, Leso V, Dolce P, Vitale R, Vetrani I, Galdi A, lavicoli I (2020) Low dose ionizing radiation exposure and risk of thyroid functional alterations in healthcare workers. Eur $\mathrm{J}$ Radiol 132:109279

6. Gould R, McFadden SL, Hughes CM (2017) Radiation dose in paediatric cardiac catheterisation: A systematic literature review. Radiography (London, England: 1995), 23(4), 358-364

7. Linder N, Sela B, German B, Davidovitch N, Kuint J, Hegesh J, Lubin D, Sack J (1997) lodine and hypothyroidism in neonates with congenital heart disease. Archives of disease in childhood. Fetal and neonatal edition 77(3):F239-F240

8. Dechant MJ, van der Werf-Grohmann N, Neumann E, Spiekerkoetter U, Stiller B, Grohmann J (2016) Thyroidal response following iodine excess for cardiac catheterisation and intervention in early infancy. Int J Cardiol 223:1014-1018 
9. Nykanen DG, Forbes TJ, Du W, Divekar AA, Reeves JH, Hagler DJ, Fagan TE, Pedra CA, Fleming GA, Khan DM, Javois AJ, Gruenstein DH, Qureshi SA, Moore PM, Wax DH (2016) CRISP: Catheterization RISk score for Pediatrics: A Report from the Congenital Cardiac Interventional Study Consortium (CCISC). Catheterization and cardiovascular interventions:, vol 87. official journal of the Society for Cardiac Angiography \& Interventions, pp 302-3092\& Congenital Cardiac Interventional Study Consortium (CCISC)

10. Vigone MC, Capalbo D, Weber G, Salerno M (2018) Mild Hypothyroidism in Childhood: Who, When, and How Should Be Treated? Journal of the Endocrine Society 2(9):1024-1039

11. Feltes TF, Bacha E, Beekman RH 3, Cheatham JP, Feinstein JA, Gomes AS, Hijazi ZM, Ing FF, de Moor M, Morrow WR, Mullins CE, Taubert KA, Zahn EM, American Heart Association (2011) American Heart Association Congenital Cardiac Defects Committee of the Council on Cardiovascular Disease in the Young, Council on Clinical Cardiology, Council on Cardiovascular Radiology and Intervention, \& Indications for cardiac catheterization and intervention in pediatric cardiac disease: a scientific statement from the American Heart Association. Circulation, 123(22), 2607-2652

12. Salerno M, Improda N, Capalbo D (2020) MANAGEMENT OF ENDOCRINE DISEASE Subclinical hypothyroidism in children. Eur J Endocrinol 183(2):R13-R28

13. Koukkou EG, Roupas ND, Markou KB (2017) Effect of excess iodine intake on thyroid on human health. Minerva Med 108(2):136-146

\section{Tables}

Table (1): Patients' characteristics ( $n=102$ patients) 


\section{Birth weight (kg), median (range)}

$3.0(1.5-4.0)$

\section{Gender}

- Male, n (\%)

- Female, n (\%)

$42(41 \%)$

$60(59 \%)$

Age at diagnosis of congenital heart disease (CHD) (months), median $4.0(0.07-30.0)$ (range)

Age at time of evaluation (months), median (range)

$13.8(1.0-36.0)$

\section{Types of CHD}

- Left to Right shunt, n (\%)

- Obstructive lesions, n (\%)

- Cyanotic, n (\%)

$60(59 \%)$

$30(29 \%)$

$12(12 \%)$

\section{CRISP ${ }^{\uparrow}$, median (range)}

$5.0(0.0-13.0)$

Number of procedures ${ }^{49}$, median (range)

$1.0(1.0-4.0)$

- One procedure, n (\%)

- Two procedures, n (\%)

$80(78 \%)$

- Three procedures, n (\%)

- Four procedures, n (\%)

$12(12 \%)$

$6(6 \%)$

$4(4 \%)$

\section{Types of cardiac catheters}

- Diagnostic Catheter, n (\%)

- Therapeutic Catheter, n (\%)

$10(10 \%)$

$92(90 \%)$

Maximum serum creatinine during exposure period, median (range)

Cumulative iodine dose (gram per $\mathrm{kg}$ ) / ( $\mathrm{ml} / \mathrm{kg}$ ), median (range)

$0.51(0.30-0.80)$

$5.29(0.75-15.0) / 17.6(2.5-$ $50)$

Duration of fluoroscopy (minutes), median (range)

$20.0(5.0-44.0)$

'CRISP: Catheterization risk score for pediatrics

${ }^{99}$ Any procedure included exposure to iodinated contrast media (surgical and/or radiological). 
Table (2): Relation between thyroid functions and cardiac catheter characteristics 
After 4 weeks

Euthyroid Hypothyroidism

$(n=90) \quad(n=12)$

CRISP', median (range)

$5.0(0.0-$

13.0)
Test of significance

$p$ value

Type of congenital heart disease

- Left to Right shunts, n (\%)

-Atrioventricular septal defect

-Ventricular septal defect

$6(6.7)$

$0(0.0)$

FET

1.0

-Atrial septal defect

8 (8.9)

$0(0.0)$

FET

0.1

-Patent ductus arteriosus

- Obstructive, n (\%)

$15(16.7) \quad 0(0.0)$

$\square^{2}=1.49$

0.221

$46(51.1) \quad 4(33.4)$

FET

0.395

-Pulmonary stenosis

-Aortic stenosis

$7(7.8)$

1 (8.3)

FET

0.643

- Cyanotic, n (\%)

$8(8.8)$

$6(50)$

FET

$0.029^{*}$

-Transposition of great arteries
$1(8.3)$

FET

0.12

Number of procedures ${ }^{99}$, median (range)

- One procedure, n (\%)

- Two procedures, n (\%)

- Three procedures, n (\%)

- Four procedures, n (\%)

\section{Types of cardiac catheters}

- Diagnostic Catheter, n (\%)

- Therapeutic Catheter, n (\%)
$10(11.1) \quad 0(0.0)$

FET

1.0

$80(88.9) \quad 12(100.0)$

Duration of fluoroscopy (minutes), median (range)

$19.5(5.0$ 44.0)

$28.0(20.0-35.0) \quad Z=2.23$
$70(77.8)$

$10(11.1) \quad 2(16.7)$

$6(6.7)$

$0(0.0)$

$4(4.4)$

$0(0.0)$
MC

0.878

$0.026^{\star}$

$5(0.75-$

15.0)/

$11(8.11-15.0) /$

$Z=2.61$

$0.007^{\star}$ 
$c^{2}$ : Chi square test, Z: Mann Whitney U test, FET: Fischer's exact test, MC: Monte Carlo test, *Significant $p$ value $<0.05$.

'CRISP: Catheterization Risk Score for Paediatrics, ${ }^{\mathbf{9}}$ All procedures which included exposure to iodinated contrast media (surgical and/or radiological).

Table (3): Univariate and multivariate analysis of predictors of hypothyroidism among the studied cases

\begin{tabular}{lllll} 
Predictors & \multicolumn{2}{l}{ Univariate analysis } & \multicolumn{2}{l}{ Multivariate analysis } \\
\cline { 2 - 5 } & $\boldsymbol{P}$ & $\mathrm{RR}(95 \% \mathrm{Cl})$ & $\boldsymbol{P}$ & $\mathrm{RR}(95 \% \mathrm{Cl})$ \\
\hline Type of cardiac lesion: & & & & \\
Aortic stenosis & $0.018^{*}$ & $10.0(1.49-66.99)$ & 0.256 & $3.76(0.38-37.13)$ \\
\hline Duration of fluoroscopy & $0.05^{*}$ & $1.12(0.99-1.26)$ & 0.121 & $1.12(0.97-1.29)$ \\
\hline Cumulative iodine dose & $0.019^{*}$ & $1.01(1.003-1.01)$ & $0.04^{*}$ & $1.00(1.00-1.01)$
\end{tabular}

RR: Relative risk, Cl: Confidence interval, *Significant $p$ value $\leq 0.05$

Table (4): Cut-off points of cumulative iodine dose and duration of fluoroscopy for detection of hypothyroidism evolution after cardiac catheterization

\begin{tabular}{llllllll} 
& AUC & $\begin{array}{l}\text { Cut off } \\
\text { point }\end{array}$ & $\begin{array}{l}\text { Sensitivity } \\
(\mathbf{9})\end{array}$ & $\begin{array}{l}\text { Specificity } \\
(\%)\end{array}$ & $\begin{array}{l}\text { PPV } \\
(\%)\end{array}$ & $\begin{array}{l}\text { NPV } \\
(\%)\end{array}$ & $\begin{array}{l}\text { Accuracy } \\
(\%)\end{array}$ \\
\hline $\begin{array}{l}\text { Cumulative iodine } \\
\text { dose (gram per kg)/ }\end{array}$ & 0.829 & $8.7 /$ & 83.3 & 65.1 & 25.0 & 96.6 & 67.3 \\
(ml per kg) & $\begin{array}{l}(0.688- \\
0.971)\end{array}$ & 26.1 & & & & & \\
\hline $\begin{array}{l}\text { Duration of } \\
\text { fluoroscopy (minutes) }\end{array}$ & $\begin{array}{l}0.780 \\
(0.632-\end{array}$ & 24 & 83.3 & 65.9 & 25.0 & 96.7 & 68.0 \\
& & & & & & \\
$0.928)$ & & & & & &
\end{tabular}

PPV: Positive predictive value, NPV: Negative predictive value, AUC: Area under curve

\section{Figures}


Figure 1

The incidence of hypothyroidism in the studied cohort at different time intervals following the cardiac catheter.

\section{ROC Curve}

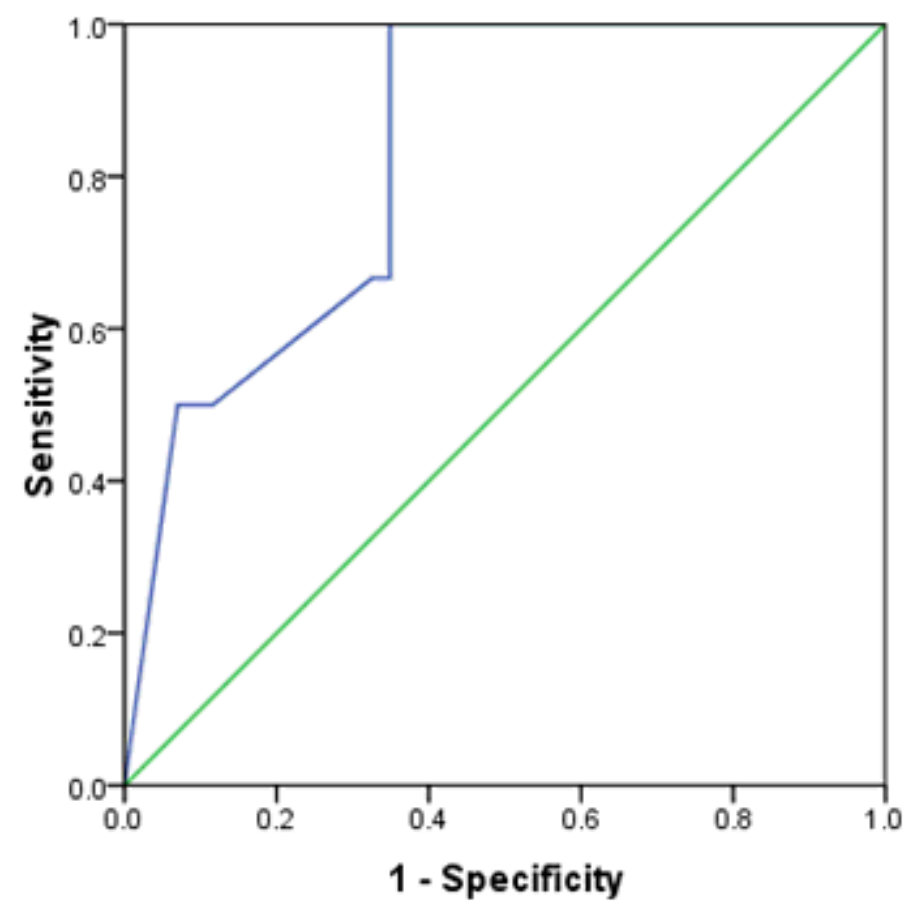

Figure 2

ROC curve for iodine dose which can predispose to hypothyroidism among the studied cases.

\section{Figure 3}

ROC curve for fluoroscopy duration which can predispose to hypothyroidism among the studied cases. 